Año 11.

Frontera

Núm. 27
Revista de Investigación

Académica sin

ISSN: 2007-8870

http://revistainvestigacionacademicasinfrontera.com

Recibido el 13 de febrero de 2018.

Dictaminado mediante arbitraje favorablemente 28 de mayo de 2018

\title{
Diagnóstico del Sistema de Costos en la Microempresa: Cinco Casos de Estudio
}

\author{
Dra. María Dolores Moreno Millanes \\ Profesor Investigador del Instituto Tecnológico de Sonora \\ mmoreno@itson.edu.mx \\ Mtro. Roberto Ruiz Pérez \\ Profesor Investigador del Instituto Tecnológico de Sonora \\ roberto.ruiz@itson.edu.mx \\ Dr. Rodolfo Valenzuela Reynaga \\ Profesor Investigador del Instituto Tecnológico de Sonora \\ rvalenzuela@itson.edu.mx
}

\section{Resumen}

En la actualidad el entorno en el cual se vienen desarrollando las empresas se ha vuelto más competitivo, ocasionando que los empresarios busquen herramientas que les permitan una supervivencia en el mercado. Parte de esa de esas herramientas son los sistemas de costos, los cuales facilitan la toma de decisiones para los administradores financieros. En esta investigación se desea elaborar un diagnóstico del sistema de costos actual a través de cinco casos de estudios con microempresas que pertenecen al sector de alimentos y bebidas. Para la recolección de información se utilizaron entrevistas estructuradas a través de un cuestionario, con los datos recabados se procedió al uso de estadística descriptiva para sismatizar e contrastar los resultados obtenidos con las teorías de costos. Los principales hallazgos detectados son: que el 76\% de los encuestados inició operaciones financiándose por recursos propios, $80 \%$ manifiestan que sus aportaciones son consideradas para mejorar 
Año 11.

Frontera

Núm. 27
Revista de Investigación

Académica sin

ISSN: 2007-8870

\title{
http://revistainvestigacionacademicasinfrontera.com
}

el sistema de costos y el 100\% fijan los precios consideran el mercado, costos de los insumos, entre otros. En donde no hubo un consenso mayoritario es la parte de las decisiones que se pudieran tomar con el sistema de costeo, ya que manifestaban que no tenían certidumbre en la asignación de los costos. Para futuras líneas de investigación se pudieran considerar otros sectores y cómo es su comportamiento.

\section{Palabras clave: Sistemas de Costos, microempresas, toma de decisiones}

\begin{abstract}
Currently the environment in which companies are developing has become more competitive, causing entrepreneurs to seek tools that allow them to survive in the market. Part of those tools are cost systems, which facilitate decision making for financial managers. In this research, it is desired to develop a diagnosis of the current cost system through five cases of studies with micro-companies that belong to the food and beverages sector. For the collection of information structured interviews were used through a questionnaire, with the data collected, proceeded to the use of descriptive statistics to systematize and contrast the results obtained with the theories of costs. The main findings are: that $76 \%$ of the respondents started operations financed by their own resources, $80 \%$ state that their contributions are considered to improve the cost system and $100 \%$ fix the prices consider the market, costs of the inputs, among others. Where there was no majority consensus is the part of the decisions that could be made with the costing system, since they stated that they had no certainty in the allocation of costs. For future lines of research, other sectors could be considered and what their behavior is like.
\end{abstract}

Keywords: Cost systems, micro-enterprises, decision making 
Año 11. Frontera

Núm. 27
Revista de Investigación Académica sin

ISSN: 2007-8870

\section{http://revistainvestigacionacademicasinfrontera.com}

\section{Introducción}

La situación económica mundial ha propiciado un ambiente en el cual se motiva cada vez más las actividades de emprendedurismo. Según Vidales, P. (2013) el término etimológico con el que se asocia la palabra emprendedor en español es el de asumir el riesgo de llevar a cabo un proyecto que es difícil y azaroso. Varias de los obstáculos observables de automotivación por desarrollar este tipo de proyectos son las condiciones macroeconómicas de los países, lo cuales, de acuerdo con Spiegel, S. (2007) pueden frenar su desarrollo económico en función del desempeño que puedan tener la inflación, la política fiscal, política monetaria, entre otras.

El análisis de acceso a oportunidades es importante ya que esto fomenta el crecimiento económico de una región. De acuerdo a publicaciones realizadas por Pineda, A. (2018) sobre el Índice Global de Emprendimiento 2018, México descendió del lugar 71 al 75 esto se debe a que registró bajas evaluaciones en los aspectos de soporte cultural, percepción de oportunidades de negocio y habilidades emprendedora. Según Aguirre, L. (2013) el estudio de estos índices son parámetros que conllevan al crecimiento económico y a la creación de empleos, los cuales son asociados como elementos clave para la competitividad y productividad de cualquier nación. Es por ello que éste artículo desea brindar a empresarios referencias sobre detección de mejoras que pudieran implementar en sus organizaciones.

En el desarrollo de la presente investigación se elaboró un diagnóstico sobre el sistema de costos que utilizan cinco microempresas que pertenecen al sector de alimentos y bebidas, así mismo se encuentran localizadas en el Sur del Estado de Sonora, específicamente en el Municipio de Cajeme. La investigación se divide en cuatro apartados, siendo el primero sobre la clasificación económica (en este punto se determina personalidad jurídica, año de operaciones, motivaciones para aperturar la empresa, entre otros); el segundo apartado es 
Año 11.

Frontera

Núm. 27
Revista de Investigación

Académica sin

ISSN: 2007-8870

\section{http://revistainvestigacionacademicasinfrontera.com}

sobre puntos importantes para determinar oportunidades de mejora del sistema de costos actual de la empresa (en este apartado se abordan la alineación de los costos con los objetivos de la empresa, si cuentan con almacén formal, mecanismos de control para materia prima, entre otros); el tercer apartado del estudio se refiere a la utilidad de la información que proporciona el sistema de costos (en este rubro se considera si hay confiabilidad en la información proporcionada por el sistema de costos, si es de utilidad para el analista financiero, si existe certidumbre en la asignación de los costos, entre otros); y finalmente el cuarto apartado de la investigación es sobre la toma de decisiones en función del sistema de costos actual (este apartado es sobre criterios de decisión para aceptación o rechazo de algún proyecto, si la empresa considera los aportes del personal para la mejora del sistema de costos actual, entre otros).

\section{Problema}

Según datos revelados por López, J. (2016) en México el 75\% de los emprendimientos fracasan debido a diversos factores, algunos de ellos son: finanzas frágiles, ausencia de objetivos y una planeación defectuosa. Es por ello que la presente investigación desea plantear un diagnóstico sobre la situación actual que presentan cinco microempresas del Sur del Estado de Sonora en cuanto a su sistema de costos actual y con ello detectar oportunidades que se pudieran traducir en fortalezas para los dueños de las organizaciones.

La relevancia de la investigación radica en que, según Arboleda, A. (2012) el manejo cuantitativo de los costos del agente económico tienen una estrecha relación interna con el uso que le puede dar la gerencia, además proporciona base para establecer objetivos y acciones operativas que se traducen en un mejor desempeño de los indicadores planteados. El uso adecuado de un sistema de costos coadyuva en la detección de problemas que pudiera tener la organización, pero su principal aportación según Roldán, Montoya, y Agudelo (2009) es la toma de decisiones que se pueden tomar en función de los resultados que arroje 
Año 11.

Frontera

Núm. 27
Revista de Investigación

Académica sin

ISSN: 2007-8870

\section{http://revistainvestigacionacademicasinfrontera.com}

el sistema los cuales van desde la optimización de los recursos monetarios, cálculos de puntos de equilibrio hasta los márgenes de utilidad.

Para fundamentar la problemática planteada en la investigación es pertinente citar a Rocha, H. (2014) el cual afirma que en México el contar con un sistema de costos adaptado a las necesidades de la organización permite elaborar un plan de mejora continua; ya que en la actualidad no se le presta la atención que se debe y a consecuencia de ello, se desconocen los beneficios que un buen sistema de costos pudiera producir. Así mismo de acuerdo con Chang, L., Nora, G., Elvira, L., y Moreno, M. (2011) han encontrado que la contabilización de costos son herramientas que permiten a los administradores generales de las entidades financieras tomar decisiones importantes sobre la planeación y control de la misma.

También se encontró evidencia sobre por qué los empresarios se muestran reacios a modificar sus sistemas de costos. En investigaciones realizadas en PyMEs de Puebla, México por Mejía, M. y Hernández, S. (2010) encontraron que una de los principales obstáculos para modificar las técnicas de contabilidad de costos y de gestión son dos: los empresarios consideran que se incrementa el trabajo del personal y que no se cuentan con especialistas del área hacia el interior de la empresa. De igual forma las autoras mencionan que mejorando esos sistemas se puede obtener información sobre la rentabilidad de los productos y la reducción de costos.

Derivado de lo anterior se plantea la siguiente pregunta de investigación: ¿En qué medida el elaborar un diagnóstico sobre el sistema de costos que tienen cinco microempresas del Sur de Sonora coadyuva en la detección de oportunidades de mejora para la organización?

\section{Objetivos}

De acuerdo con el portal PYMEX (2012), las organizaciones deben de fijarse objetivos para lograr resultados alcanzables, o bien, para vislumbrar hacia dónde se procura llegar. Tener 
Año 11.

Frontera

Núm. 27
Revista de Investigación

Académica sin

ISSN: 2007-8870

\section{http://revistainvestigacionacademicasinfrontera.com}

objetivos se considera vital para obtener éxito en un negocio, ya que son los que determinan el curso de la organización y motivan a los miembros de la empresa a lograrlos. Es por ello que la presente investigación persigue un objetivo general y varios metodológicos.

\section{Objetivo general:}

Determinar en qué medida la elaboración de un diagnóstico sobre el sistema de costos de cinco microempresas del Sur de Sonora coadyuva en la detección de oportunidades de mejora para la organización.

Objetivos metodológicos:

a) Elaboración del contexto general en el cual se desarrolla el fenómeno.

b) Elaboración de un marco conceptual del fenómeno.

c) Elaboración de un instrumento de medición.

d) Definición de la población (casos de estudio).

e) Análisis e interpretación de los resultados obtenidos.

\section{Desarrollo}

Para continuar con el desarrollo es necesario elaborar un marco teórico el cual surge a partir de las variables objeto de estudio las cuales son: clasificación económica e inicio de operaciones, diagnóstico sobre oportunidades del sistema de costos actual, utilidad de la información que proporciona el sistema de costos actuales y toma de decisiones en función del sistema de costos actuales. Empezaremos analizando la primera variable.

La clasificación económica e inicio de operaciones, estas variables son importantes porque ayudan a determinar cuál es la personalidad jurídica de las organizaciones, así como la madurez de la misma. De acuerdo con Bautista, G. (2015) las empresas se pueden de tres formas diferentes: la primera es derivada de su giro o actividad (empresas industriales, comerciales y de servicios); la segunda es la que tiene que ver con el origen de su capital 
Año 11.

Frontera

Núm. 27
Revista de Investigación

Académica sin

ISSN: 2007-8870

\section{http://revistainvestigacionacademicasinfrontera.com}

(pueden ser públicas o privadas); y el tercer tipo va de acuerdo a su magnitud como chica, mediana y grande (aquí se consideras criterios financieros, el personal ocupado, la producción, los niveles de venta, entre otros).

Para efectos de la presente investigación nos enfocaremos en los microempresarios, los cuales según la Secretaría de Economía (2010) los clasifica como aquellas entidades económicas que cuentan con menos de 10 empleados y que obtienen ingresos por ventas hasta por 4 millones de pesos; este tipo de organizaciones constituyen el $95 \%$ del total de las empresas formalmente registradas en México, generan el 45.6\% del empleo y contribuyen con un $15 \%$ al Producto Interno Bruto, es por ello que es importante su estudio.

Otra clasificación es la proporcionada por los Censos Económicos (2009) publicado por el INEGI en 2011 en el cual cita a la Microindustria como empresas que requieren de hasta 15 personas y los ingresos por concepto de ventas netas está topada hasta 30 millones de pesos al año. Cómo se puede observar la clasificación del tipo de empresa varía según el organismo que publica. Para efectos de la investigación tomaremos este último criterio presentado por el INEGI.

Así mismo también se estudia la madurez de las empresas, ya que en publicaciones realizadas por Durán, C. (2014) demuestra que una empresa que cuenta con un número mayor de años en el mercado trae una gama de atributos considerados por los consumidores como lo es: el prestigio, la reputación, respaldo de los proveedores, experiencia, estabilidad, entre otros los cuales pueden generar una ventaja competitiva sobre empresas jóvenes.

La segunda variable tiene que ver sobre la importancia de un diagnóstico sobre oportunidades del sistema de costos actual, para ello es conveniente mencionar que existen formas para clasificar los costos. Según Salas, A. (2013) menciona que hay varias formas de clasificar los 
Año 11.

Frontera

Núm. 27
Revista de Investigación

Académica sin

ISSN: 2007-8870

\section{http://revistainvestigacionacademicasinfrontera.com}

costos, algunas de ellas son: las que van en función del área donde se consume (costos de producción, administración, distribución y financiamiento); según su identificación (directos o indirectos); de acuerdo al momento en que se calcula (históricos o predeterminados); de acuerdo con el momento en el que se reflejan en el estados de resultados (costo del período o costo del producto); De acuerdo con el control que se tenga sobre su consumo (controlables o no controlables); y el más importante, el que va de acuerdo a su comportamiento (costos fijos, costos variables, costo semi variables, mixtos y escalonados).

Ya que se tiene la información sistematizada sobre el origen de los costos, es conveniente establecer un sistema adecuado a las necesidades específicas de la organización, para con ello obtener beneficios económicos. De acuerdo con Ríos, M. (2014) citando a Kaplan y Cooper (1990) menciona que los sistemas de costos: deben de estar en posibilidades para plantear que nuevos productos o servicios se ofertarán, brindar áreas de oportunidad para la mejora continua o reingeniería, facilitar decisiones relacionadas con inversiones y determinación de los precios, facilidad para seleccionar los servicios de proveeduría, optimización de procesos, generar un poder de negociación con clientes, precio, calidad, entre otros.

La tercera variable es la utilidad de la información que proporciona el sistema de costos, la cual menciona el Consultorio Contable EAFIT (2007) que para que el sistema de costos sea eficiente es necesario realizar una asignación correcta de los costos, con ello se permite que con el precio de venta establecido se obtenga un margen de contribución real aceptable, así mismo indica que la parte más compleja de la asignación de costos, tiene que ver con el prorrateo de los costos indirectos de fabricación, ya que en muchas ocasiones se hacen asignaciones arbitrarias lo cual no muestra el costo real de los productos. Es por ello que el 
Año 11.

Frontera

Núm. 27
Revista de Investigación

Académica sin

ISSN: 2007-8870

\section{http://revistainvestigacionacademicasinfrontera.com}

sistema de costos nos debe de proporcionar información fidedigna para poder realmente tomar decisiones adecuadas a las problemáticas de la empresa.

La cuarta variable es sobre utilidad de la información que proporciona el sistema de costos actuales y toma de decisiones en función del sistema de costos actuales. Según Aravena, M., Fara, C., Torres, V., Verde, N., y Arcuch, G. (2012) el sistema de costos desea clarificar la forma en la cual ingresa el flujo de efectivo de la empresa para con ello determinar cuáles productos o servicios son los que ofrecen mayores ganancias y por otro lado saber si la estructura de costos de la empresa está en posibilidad de tener sustentabilidad económica.

Así mismo el Consultorio Contable EAFIT (2007) el conocimiento sobre los costos pudiera transformarse bajo ciertas circunstancias en una ventaja competitiva para las empresas, ya que el saber cuánto en realidad le está costando producir un producto o servicio, le permite tomar decisiones sobre si puede renegociar con su proveedor o bien optar por otro ya sea nacional o internacional. Es decir el administrador financiero puede tomar decisiones estratégicas de compras y líneas de producción.

Metodología de la Investigación: En este apartado se presentan los enfoques que se utilizaron para el desarrollo de la investigación, las fuentes de dónde se elaboró el instrumento de medición así como la caracterización de la población que fue elegida para la elaboración de los casos de estudio y el método de análisis para los resultados obtenidos. La investigación tiene dos enfoques el cualitativo y cuantitativo, predominando el cualitativa, ya que en esta fase del proceso se encuentra en la elaboración de un diagnostico que posteriormente se puede transformar en un insumo para hacer de las debilidades con las que cuenta fortalezas. El estudio es exploratorio, ya que se trabaja sobre brechas del conocimiento que tienen diferentes resultados según el contexto; es descriptivo porque se muestran resultados específicos de las microempresas. La investigación es no experimental, ya que no se tiene 
Año 11.

Frontera

Núm. 27
Revista de Investigación

Académica sin

ISSN: 2007-8870

\section{http://revistainvestigacionacademicasinfrontera.com}

control sobre las variables. Así mismo se utilizaron técnicas documentales para el proceso de investigación del problema y técnica de campo, ya que se acudió directamente al lugar físico donde se viene desenvolviendo el fenómeno objeto de estudio.

Población: La población es finita, ya que solo se realizaron cinco casos de estudio. El tipo de instrumento empleado fue un cuestionario con treinta y siete reactivos, los cuales fueron adaptados de: la Encuesta Nacional sobre Productividad y Competitividad de las Empresas ENAPROCE 2015 para microempresas la cual es emitida por el INEGI; también del artículo de Ríos, M. (2014) sobre "Método de Diagnóstico para Determinar el Sistema de Costes en una Pyme”; y del libro de Del Río, C., Del Río, C. y Del Río, R. (2011) Costos I.

Método de Análisis: El método de análisis fue a través de la elaboración de los casos de estudio, empleado estadística descriptiva, la cual según Mancilla, H. y Parra, J. (2013) son estudios en los cuales se obtiene información, se sistematiza, presenta y se transforma en números para su análisis.

\section{Resultados}

Dentro de los principales resultados obtenidos del primer apartado del instrumento empleado sobre la clasificación económica e inicio de operaciones se desprende que la personalidad jurídica de los encuestados se distribuye de la siguiente manera: el $80 \%$ están registrados como personas físicas y el $20 \%$ como personas morales. Así mismo los principales productos que fabrican son se muestran en la siguiente gráfica: 
(Enero- junio 2018)

"El saber de mis hijos
hará mi grandeza"

Revista de Investigación

Año 11.

Académica sin

Frontera

ISSN: 2007-8870

Núm. 27

\section{http://revistainvestigacionacademicasinfrontera.com}

Figura 1. Principal actividad económica.

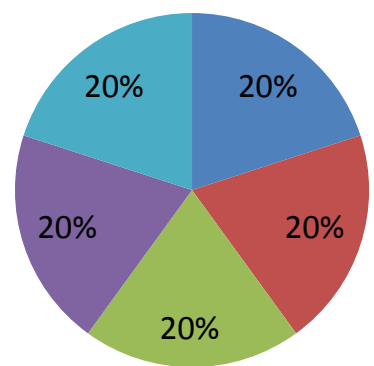

Transformar y vender alimentos

Elaboracion de tortillas de harina

Venta de tacos

Elaboracion de cafe

Fuente: Elaboración propia, 2018

En cuanto a las cuestiones de género se puede detectar que los microempresarios tienen un sesgo hacia la contratación personal femenino, ya que del 100\% de la población analizada sólo el $36 \%$ corresponde al género masculino, lo resultados se muestran en la siguiente tabla:

Figura 2. Sexo de los Empleados

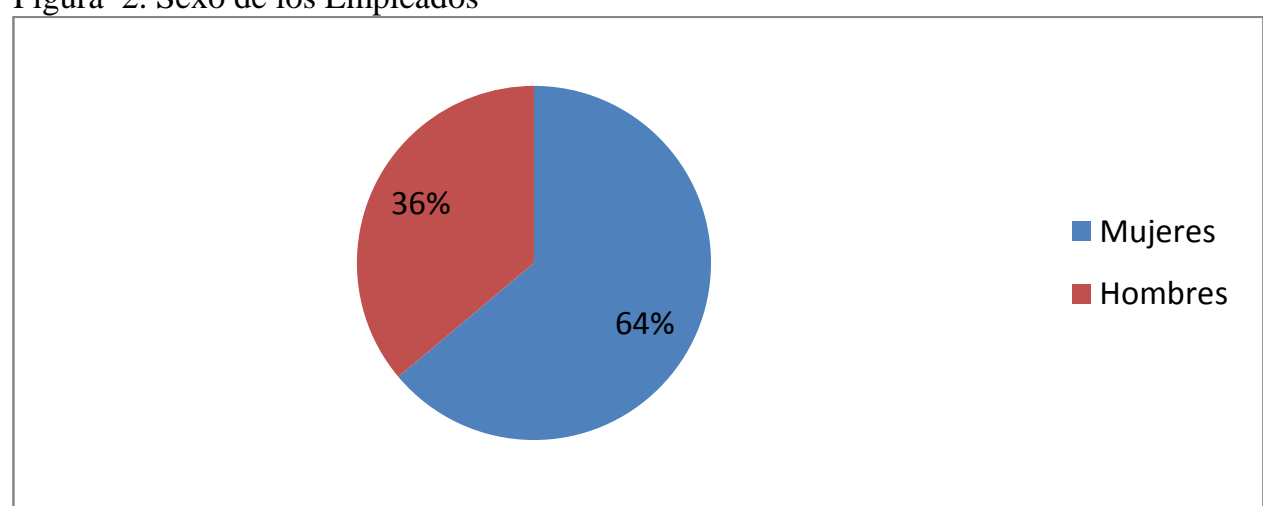

Fuente: Elaboración propia, 2018 
Año 11.

Frontera

Núm. 27
Revista de Investigación

Académica sin

ISSN: 2007-8870

\section{http://revistainvestigacionacademicasinfrontera.com}

En cuanto al inicio de operaciones, se detectó que la mayor concentración respecto a la apertura del negocio son empresas de reciente creación con un 40\% e iniciaron en el 2017 , un $20 \%$ cuenta con tres años de experiencia, otro $20 \%$ cuenta con más de cinco años y la empresa más longeva cuenta con diecisiete años de experiencia. En cuanto a la obtención de los recursos financieros para el inicio de la empresa, se encontró que en su mayoría se obtienen de recursos propios, a continuación se muestran los resultados:

Figura 3. Obtención de la Inversión Inicial

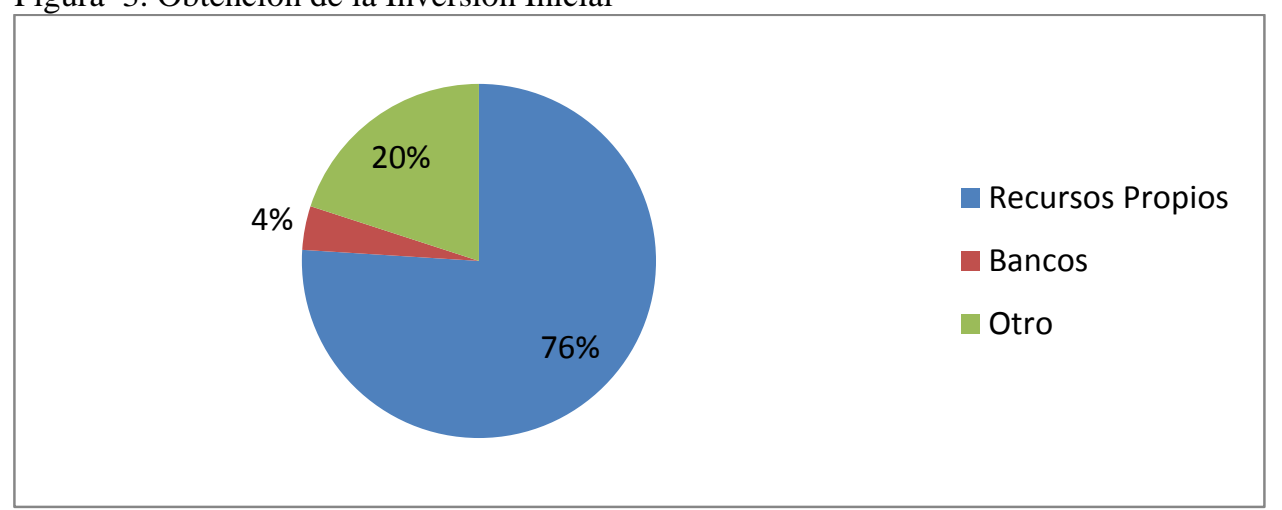

Fuente: Elaboración propia, 2018.

En cuanto a las razones por las cuales decidieron aperturar la empresa, de las nueve opciones disponibles, sólo utilizaron dos las cuales se muestran en la siguiente gráfica: 


\section{Revista de Investigación}

Año 11.

Académica sin

Frontera

ISSN: 2007-8870

\section{http://revistainvestigacionacademicasinfrontera.com}

Figura 4. Motivación para la apertura de la empresa

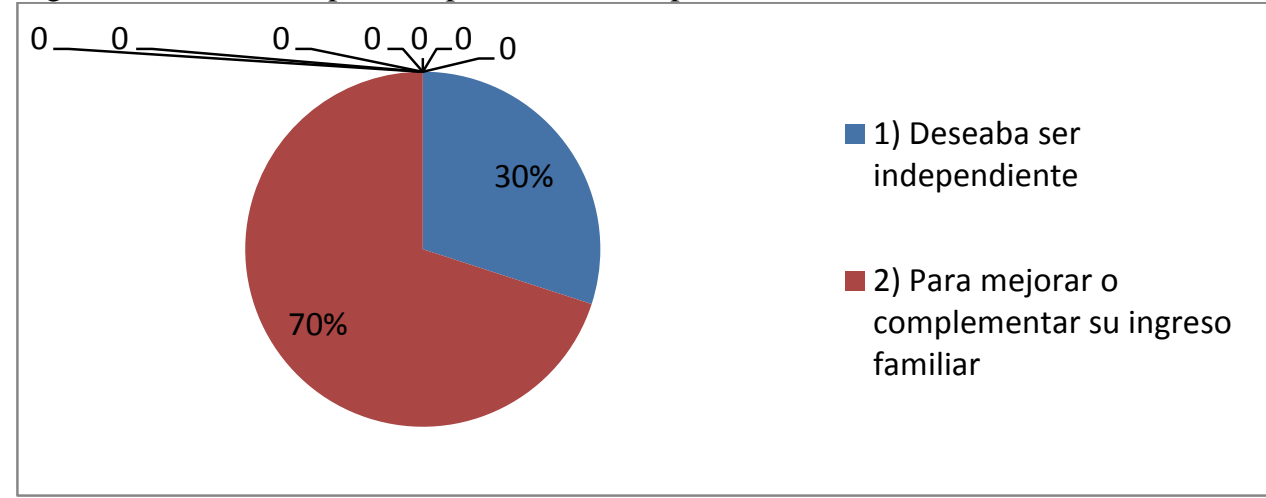

Fuente: Elaboración propia, 2018

En cuanto a la sección II del instrumento de evaluación se encontró que el $60 \%$ de los encuestados considera que siempre: se incrementan cada año el número de productos debido a la visión de la empresa, se incrementan cada año los gastos indirectos y que los gastos generales van alineados con los objetivos de la empresa; $80 \%$ de los encuestados considera que siempre: la empresa es competitiva con los productos que ofrece, que los empleados que realizan más de dos funciones son más eficientes y qué en los últimos dos años se han mejorado políticas, procedimientos y exigencias de cada departamento; $100 \%$ de los encuestados consideran que siempre: los precios de los productos se determinan en función de: mercado, costos de insumos, experiencia de la empresa, competidores y situación económica; $40 \%$ consideran que siempre: los empleados realizan más de una actividad; $40 \%$ consideran la opción de Nunca y A veces se invierte en tecnología para el reemplazo de mano de obra. En la siguiente tabla 1 se muestran los resultados obtenidos: 
Año 11.

Frontera

Núm. 27
Académica sin

ISSN: 2007-8870

\section{http://revistainvestigacionacademicasinfrontera.com}

Tabla 1. Diagnóstico sobre el sistema de costos actual

\begin{tabular}{|c|c|c|c|c|c|}
\hline Ítems & Nunca & $\begin{array}{l}\text { Casi } \\
\text { Nunca }\end{array}$ & A veces & $\begin{array}{l}\text { Casi } \\
\text { Siempre }\end{array}$ & Siempre \\
\hline $\begin{array}{l}\text { Según la visión de la empresa, cada año se } \\
\text { incrementan el número de productos }\end{array}$ & & $20 \%$ & & $20 \%$ & $60 \%$ \\
\hline $\begin{array}{l}\text { Los gastos indirectos se van incrementando } \\
\text { cada año }\end{array}$ & & & $20 \%$ & $20 \%$ & $60 \%$ \\
\hline $\begin{array}{l}\text { Los gastos generales van alineados con los } \\
\text { objetivos de la empresa }\end{array}$ & & & $20 \%$ & $20 \%$ & $60 \%$ \\
\hline La empresa es competitiva con sus productos & & & $20 \%$ & & $80 \%$ \\
\hline $\begin{array}{l}\text { Los precios de los productos se determinan en } \\
\text { función de: Mercado, costos de insumos, } \\
\text { experiencia de la empresa, competidores y } \\
\text { situación económica. }\end{array}$ & & & & & $100 \%$ \\
\hline $\begin{array}{l}\text { Se invierte en tecnología para el reemplazo de } \\
\text { mano de obra }\end{array}$ & $40 \%$ & & $40 \%$ & $20 \%$ & \\
\hline $\begin{array}{l}\text { Los empleados realizan funciones diversas } \\
\text { para ser más eficientes }\end{array}$ & & & $20 \%$ & & $80 \%$ \\
\hline $\begin{array}{l}\text { Los sueldos y prestaciones del personal } \\
\text { representan un porcentaje alto de los costos } \\
\text { controlables }\end{array}$ & & & $20 \%$ & $20 \%$ & $60 \%$ \\
\hline $\begin{array}{l}\text { En la empresa un empleado realiza más de una } \\
\text { actividad diferente }\end{array}$ & $20 \%$ & $20 \%$ & & $20 \%$ & $40 \%$ \\
\hline $\begin{array}{l}\text { En los últimos dos años se han mejorado } \\
\text { políticas, procedimientos y exigencias de cada } \\
\text { departamento }\end{array}$ & $20 \%$ & & & & $80 \%$ \\
\hline
\end{tabular}

También se detectó que el 100\% de los encuestados cuenta con un espacio físico para el almacén y el $80 \%$ lleva un control de la materia prima. De los que si llevan control el $50 \%$ utiliza solicitudes compra y el otro $50 \%$ utiliza formas de pedido para llevar un control de la materia prima. Así mismo se detectó que el $80 \%$ de la población estudiada no tiene contemplada una cantidad mínima de recompra de inventario de materia prima. Para estimar 
Año 11.

Frontera

Núm. 27
Revista de Investigación

Académica sin

ISSN: 2007-8870

\section{http://revistainvestigacionacademicasinfrontera.com}

el componente de Gastos Indirectos de Fabricación (GIF) el desembolso más representativo es la renta, a continuación se muestran los resultados:

Figura 5. Gastos Indirectos de Fabricación

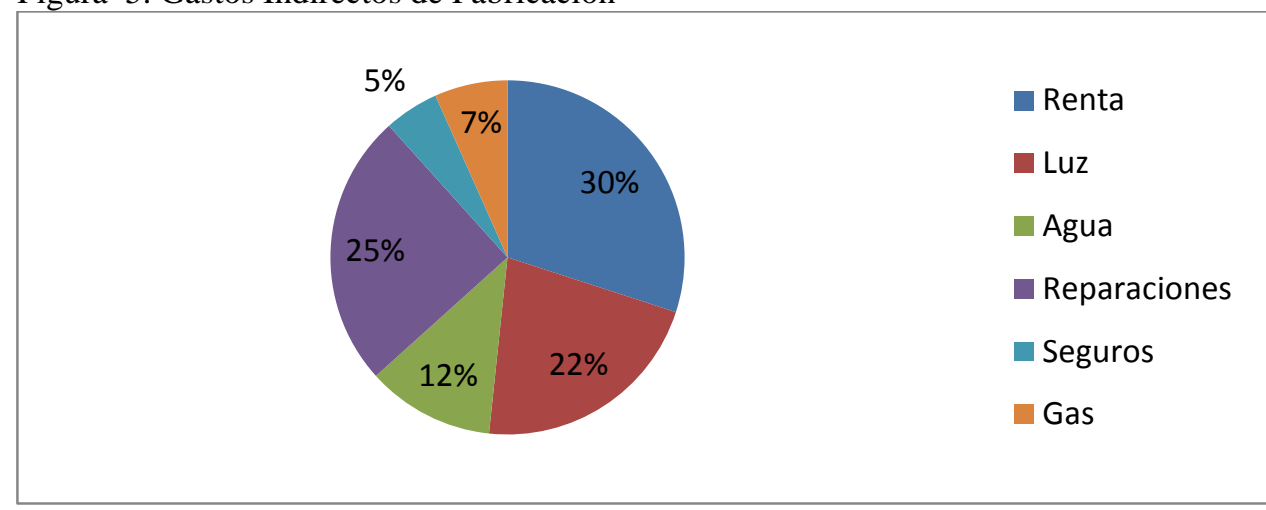

Fuente: Elaboración propia, 2018.

Continuando con los resultados se encontró que el $40 \%$ de los empresarios encuestados no tiene asignado formalmente la parte proporcional de GIF a cada producto que vende. Así mismo se detectó que el $60 \%$ de las empresas cuenta con un organigrama formal. Otro punto importante que se descubrió con la investigación es que el $60 \%$ de las empresas no cuenta con una descripción detallada de cada uno de los puestos que desempeñan cada uno de los empleados del área de producción. Finalmente el formato que más utilizan para el control de mano de obra es la nómina o lista de raya con un $71 \%$, a continuación se muestran los resultados: 
Año 11.

Frontera

Núm. 27
Académica sin

ISSN: 2007-8870

\section{http://revistainvestigacionacademicasinfrontera.com}

Figura 6. Formatos para Control de Mano de Obra

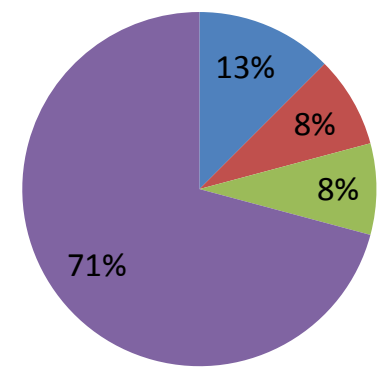

Registro de personal

- Tarjeta de asistencia

- Tiempo Extra

- Nómina o Lista de raya

Fuente: Elaboración propia, 2018.

Los resultados de la tercera sección del instrumento se descubrió que: el $60 \%$ de los encuestados considera que siempre pueden detectar problemas con el sistema de costos actual; $40 \%$ piensan que siempre se puede tener confianza en la veracidad de los reportes que genera el sistema de costeo actual, certidumbre en la asignación de los costos, contribuye en el desarrollo de innovaciones y nuevos productos, el analistas de costos puede tomar decisiones y es de utilidad para la organización; un 40\% considera que casi siempre el sistema de costos actual contribuye en el desarrollo de innovaciones y nuevos productos; un $40 \%$ considera que con el sistema actual, A veces se pueden detectar problemas y finalmente el $40 \%$ de los encuestados considera que nunca tienen certidumbre en la asignación de los costos. El resto de los resultados se presentan en la tabla 2: 
Año 11.

Frontera

Núm. 27
Académica sin

ISSN: 2007-8870

\section{http://revistainvestigacionacademicasinfrontera.com}

Tabla 2. Utilidad de la Información del Sistema de Costos Actual

\begin{tabular}{|c|c|c|c|}
\hline & Nunca Casi Nunca & A Veces & Casi Siempre \\
\hline $\begin{array}{l}\text { Confianza en la veracidad de los reportes del sistema de costos } \\
\text { actual }\end{array}$ & $20 \%$ & $20 \%$ & $20 \%$ \\
\hline Certidumbre en la asignación de los costos & $40 \%$ & & $20 \%$ \\
\hline $\begin{array}{l}\text { Con el sistema de costes actual, se pueden desarrollar } \\
\text { innovaciones y diseño de nuevos productos }\end{array}$ & & $20 \%$ & $40 \%$ \\
\hline El analista de costos puede tomar decisiones & & $60 \%$ & \\
\hline Con el sistema actual se pueden detectar problemas & & $40 \%$ & \\
\hline Con el sistema actual se considera que es de utilidad & $20 \%$ & $20 \%$ & $20 \%$ \\
\hline
\end{tabular}

Fuente: Elaboración propia, 2018.

Continuando con la investigación, llegamos a la cuarta sección, la cual trata sobre las decisiones que los microempresarios pudieran tomar en función del sistema actual de costos. El $60 \%$ de los encuestados considera que A veces la empresa considera los aportes del personal de ventas para la mejora del sistema de costos; El 40\% considera que A veces el administrador financiero de la empresa toma decisiones basadas en el sistema de costos actual y que el gerente general acepta o rechaza proyectos con la información que arroja el sistema de costos; de igual forma el $40 \%$ de los encuestados considera que Siempre el administrador financiero de la empresa toma decisiones basadas en el sistema de costos actual y que el gerente general acepta o rechaza proyectos con la información que arroja el sistema de costos. El resto de los resultados se muestran en la tabla 3: 


\section{Revista de Investigación}

Año 11.

Académica sin

Frontera

Núm. 27

ISSN: 2007-8870

\section{http://revistainvestigacionacademicasinfrontera.com}

Tabla 3. Toma de decisiones en función del sistema actual de costos

\begin{tabular}{|c|c|c|c|c|}
\hline & Nunca & Casi Nunca & A Veces & Casi $\mathrm{Si}$ \\
\hline $\begin{array}{l}\text { El administrador financiero de la empresa, toma decisiones basadas en } \\
\text { el sistema de costos actual }\end{array}$ & $20 \%$ & & $40 \%$ & \\
\hline $\begin{array}{l}\text { La empresa considera los aportes del personal del área de ventas para } \\
\text { la mejora del sistema de costos }\end{array}$ & $20 \%$ & & $60 \%$ & \\
\hline $\begin{array}{l}\text { El gerente general de la empresa considera la información que arroja } \\
\text { el sistema de costos para la aceptación o rechazo de proyectos. }\end{array}$ & $20 \%$ & & $40 \%$ & \\
\hline
\end{tabular}

Fuente: Elaboración propia, 2018.

\section{Conclusiones y Recomendaciones}

En función de los resultados obtenidos se puede decir que el tipo de personalidad jurídica que poseen los microempresarios analizados es el de personas físicas con un $80 \%$, esto concuerda con lo planteado por Galeano (2015) respecto a que: es un esquema fácil, el propietario es el único dueño del negocio y le permite tomar decisiones con rapidez, el capital de inversión no está sujeto a una cantidad limitada y se puede obtener de recursos propios o préstamos, entre otras características que superan a la personalidad jurídica de personas morales.

En cuanto a la perspectiva de género se encontró que el género femenino predomina ya que el $64 \%$ de los empleados son mujeres; éste hallazgo concuerda con los resultados planteados por los Censos Económicos (2009) del Instituto Nacional de Estadística y Geografía donde publicaron que en el año de $200853.4 \%$ de los empleados en el sector de alimentos y bebidas al por menor son mujeres. Aunque existe una brecha superior al $10 \%$ tiene consistencia respecto a la mayoría de género.

En el apartado sobre cómo fue la estrategia de financiamiento se encontró que el $76 \%$ inicio operaciones utilizando recursos propios, sólo el $4 \%$ pudo obtener préstamos bancarios y el $20 \%$ recurrió a otros métodos de financiamiento. Este resultado es similar al presentado por 
Año 11. Frontera

Núm. 27
Revista de Investigación Académica sin

ISSN: 2007-8870

\section{http://revistainvestigacionacademicasinfrontera.com}

Vivanco, N. (2014) donde demuestran que el 75\% de los empresarios recurrieron a fuentes propias de financiamiento para cumplir su objetivo de inicio de operaciones, sólo el $9 \%$ obtuvo préstamos bancarios y el resto fue de otras alternativas. Algunas de las causas que originan este tipo de financiamiento se deben a que por lo general los emprendedores son jóvenes con poco historial crediticio, así como el hecho de que sus negocios en sus inicios eran un tanto informales.

Respecto al por qué se decidieron por iniciarse como emprendedores y aperturar su negocio se encontró que el $70 \%$ lo hizo para mejorar o complementar su ingreso familiar, este resultado concuerda con el planteado por Medina (1998) el cual indica que un indicador de bienestar económico es el ingreso real que se percibe. Este resultado se complementa debido a que en la actualidad el valor del dinero ha caído precipitosamente como consecuencia de los efectos inflacionarios, la canasta básica y los principales servicios se han encarecido en mayor proporción que el incremento salarial real.

Un hallazgo interesante detectado es que el número de productos a producir depende directamente de la visión que tenga la empresa (60\% respondió que Siempre es así), en eso se basa su incremento o no, esto se puede complementar con lo planteado con Matarranz, A. (2014) el cual menciona que la visión de los productos debe de ir enfocada a un mediano y largo plaza, para establecer cuáles son lo que nuestros futuros clientes desean tener, así como la satisfacción que les dará. Así mismo un 60\% opina que los gastos indirectos se incrementan cada año y que el desembolso en gastos generales van alineados con los objetivos de la empresa. Éste incremento corresponde a lo planteado por Díaz, M. (2006) que debido a la naturaleza de estas erogaciones conforma pasa el tiempo se van encareciendo, además poseen una relación directa con la producción, es decir, si se produce más se incrementan los 
Año 11.

Frontera

Núm. 27
Revista de Investigación

Académica sin

ISSN: 2007-8870

\section{http://revistainvestigacionacademicasinfrontera.com}

mismos, una manera para generar ahorros es a través de innovaciones tecnológicas que modifiquen los procesos.

Otro punto sobresaliente fue la opinión de los micro empresarios respecto a que ellos consideran que la empresa es competitiva con los productos que ofrece, que los empleados que realizan más de dos funciones son más eficientes y que qué en los últimos dos años se han mejorado políticas, procedimientos y exigencias de cada departamento (80\% de la población lo manifestó de esa manera). Un punto no favorable respecto a estas prácticas es el que tiene que ver con la realización de más de una actividad dentro de la empresa, ya que según Vargas, I. (2010) no garantiza la calidad, y adicionalmente puede ocasionar daños a la salud, por lo cual es conveniente establecer una jerarquía de prioridades para poder obtener un desempeño laboral.

De igual forma, el 100\% de los encuestados consideran para fijar los precios de los productos que ofertan al mercado se debe de considerar la situación del mercado, los costos de insumos, la experiencia de la empresa, los competidores y la situación económica por la cual atraviesa el país. Un punto sobresaliente donde no se detectó evidencia significativa fue respecto a la inversión en tecnología para el reemplazo de mano de obra, esto tal vez se debe a que las operaciones que realizan los empleados requieren de uso intensivo de fuerza laboral física la cual a tiende a clientes directamente, por eso su reemplazo es más complicado; sin embargo según Muñoz, M. (2017) en México existe una tendencia hacia la automatización, lo que representa un potencial del $52 \%$ de empleos que pueden ser sustiuidos por tecnología (robots o maquinaria). En estadísticas reales serían alrededor de 25.5 millones de 49.3 millones que se tienen registrado (esto significa que uno de cada dos empleos pudiera ser sustituido).

También se encontró que todos los empresarios cuentan con almacén físico para las materias primas, sin embargo, un $20 \%$ no lleva un control formal del mismo; siendo las solicitudes de 
Año 11. Frontera

Núm. 27
Revista de Investigación Académica sin

ISSN: 2007-8870

\section{http://revistainvestigacionacademicasinfrontera.com}

compra y formas de pedido las más empleadas. Un punto de alerta es el hecho de que el $80 \%$ de la población estudiada no tiene estipulado formalmente una cantidad mínima de recompra de inventario de materia prima, esto se contrapone con lo planteado por Espinoza, M. (2008) ya que en sus publicaciones recomienda que se tenga niveles correctos de mercancía mínimos y máximos para incrementar los beneficios, y no tener problemas al momento de querer surtir las mercancías con los proveedores. En cuanto a los Gastos Indirectos de Fabricación (GIF) los más representativos son la renta, la luz y las reparaciones (abarcando el $77 \%$ en importancia).

Otra área de oportunidad detectada es el hecho de que sólo el $60 \%$ de los empresarios tienen formalmente prorateados los GIF a cada producto que vende; también es necesario que todos cuenten con un organigrama formal (hay un $40 \%$ que no cuenta con el). De igual forma el $60 \%$ manifiesta que no cuenta con una descripción detallada del perfilamiento del puesto de producción, lo cual representa un foco rojo, ya que según la Secretaría de las Funciones Públicas de México en su Dirección General de Desarrollo Humano y Servicio Profesional de Carrera. (s/f) establece que el perfilamiento de puestos logra detectar las aptitudes, cualidades y capacidades que, se consideran esenciales para el quehacer y desempeño del trabajo. También se encontró que el formato que más emplean para llevar a cabo del el control de mano de obra es la nómina o lista de raya con un $71 \%$ de preferencia.

Respecto a la utilidad de la información del sistema de costos actual sólo el $60 \%$ considera que se encuentra en capacidad de detectar problemas a los que se enfrenta la empresa utilizando el sistema de costos actual; así mismo el 60\% considera que sólo a veces la empresa considera los aportes del personal del área de ventas para la mejora del sistema de costos. Respecto a los otros indicadores no hay claridad en los empresarios, ya que ninguna de las opciones tuvo una mayoría significativa respecto a las otras opciones, es decir no tienen 
Año 11.

Frontera

Núm. 27
Revista de Investigación

Académica sin

ISSN: 2007-8870

\section{http://revistainvestigacionacademicasinfrontera.com}

confianza en la veracidad de los reportes del sistema de costeo, no hay total claridad en la asignación de los costos y en cuanto a la utilidad que puede tener el sistema de costos para la organización. Estos resultados tienen un área importante de oportunidad, ya que según Cruz, M. (s/f) la correcta contabilidad de un sistema de costos ofrece oportunidades de desarrollarse a las empresas haciéndolas más rentables antes sus competidores, tomando decisiones que optimicen el patrimonio.

En cuanto a las decisiones que los microempresarios pudieran tomar en función del sistema actual de costos se encontró evidencia mayoritaria respecto a que sólo a veces (con un $60 \%$ de los encuestados) consideran la empresa considera los aportes del personal de ventas para la mejora del sistema de costos; el resto de los apartados no cuentan con mayoría de tal forma que no se tiene certeza las decisiones que puede tomar el administrador financiero de la empresa y la forma en la cual el gerente general aceptará o rechazará los proyectos utilizando la información del sistemas de costos. Esto representa una debilidad para las empresas analizadas, ya que según Ríos, M. (2014) "Cualquier empresa requiere de un sistema de costes que le apoye con información veraz y oportuna que le permita desarrollar estrategias para un crecimiento sostenido y competitivo" lo cual no está sucediendo en la presente investigación.

Para concluir se puede afirmar que aplicar un diagnóstico para detectar áreas de oportunidad que se pueden presentar en un sistema de costos puede atraer múltiples beneficios para las organizaciones, entre los más destacados es la optimización de los recursos lo cual las volverá más competitivas y prósperas. Para futuras líneas de investigación se pudieran tomar sectores específicos de alguna región ampliando la muestra, con ello se lograría identificar y apoyar a las organizaciones a que logren permanecer más en el mercado, para que de esa forma se establezcan bases para el desarrollo económico y social de las entidades. 
Año 11.

Frontera

Núm. 27
Revista de Investigación

Académica sin

ISSN: 2007-8870

\section{http://revistainvestigacionacademicasinfrontera.com}

\section{Referencias Bibliográficas}

Aguirre, L. (2013). Potencializando el emprendedurismo y su impacto económico. 06 de marzo de 2018, de Forbes México Sitio web: https://www.forbes.com.mx/potencializando-el-emprendedurismo-y-su-impactoeconomico/

Aravena, M., Fara, C., Torres, V., Verde, N., y Arcuch, G. (2012). Creación de una herramienta de diagnóstico para la Micro y Pequeña Empresa(Doctoral dissertation, Tesis de Maestría, Universidad de Chile). Recuperado de: http://repositorio. uchile. cl/handle/2250/108118).

Arboleda, A. (2012). Importancia de los Sistemas de costos. 01 marzo 2018, de Andres Marketing Sitio web: https://andresmarketing.blogspot.mx/2012/02/importancia-delos-sistemas-de-costos.html

Bautista, G. B. (2015). Clasificación de las empresas en México. Vida Científica Boletín de la Escuela Preparatoria No. 4, 3(5).

Censos Económicos (2009). Los hombres y las mujeres en las actividades económicas: Censos Económicos 2009 / Instituto Nacional de Estadística y Geografía.-- México : INEGI, c2011

Chang, L., Nora, G., Elvira, L., \& Moreno, M.(2011). LA IMPORTANCIA DE LA CONTABILIDAD DE COSTOS. Instituto Tecnológico de Sonora, 17.

Cruz, M. (s/f). La contabilidad de costos y su importancia para las Pymes. 02 febrero de 2018, de OpenPyme México Sitio web: https://www.openpyme.mx/es/contenido/lacontabilidad-de-costos-y-su-importancia-para-las-pymes

Consultorio Contable EAFIT. (2007). La Importancia de Conocer y saber Clasificar los Costos para una buena toma de Decisiones. 02 febrero 2018, de Universidad EAFIT 
Año 11.

Frontera

Núm. 27
Revista de Investigación

Académica sin

ISSN: 2007-8870

\section{http://revistainvestigacionacademicasinfrontera.com}

Sitio web: http://www.eafit.edu.co/escuelas/administracion/consultoriocontable/Documents/boletines/costos-presupuesto/b11.pdf

Del Río, C., Del Río, C. y Del Río, R. (2011). III. En Costos I Históricos (354). México: Cengage Learning.

Díaz, M. (2006). Acumulación de los costos. 02 de febrero de 2018, de mailxmail.com Sitio web: http://www.mailxmail.com/curso-acumulacion-costos/costos-indirectosfabricacion

Dirección General de Desarrollo Humano y Servicio Profesional de Carrera. (s/f). ¿QUÉ ES EL PERFIL DEL PUESTO? . 02 de febrero de 2018, de Secretaría de la Función Pública, México Sitio web: http://www.usp.funcionpublica.gob.mx/manuales/manualesIngreso/documentos/Quee selperfildelpuesto.pdf

Durán, C. (2014). Madurez de un negocio: ¿riesgo o ventaja competitiva?. 05 marzo 2018, de Forbes México Sitio web: https://www.forbes.com.mx/madurez-de-un-negocioriesgo-o-ventaja-competitiva/

Espinoza, M. (2008). Aprovisionamiento y control de productos y materiales.. 02 febrero 2018, de Escuela Nacional de Hotelería, República Dominicana Sitio web: http://enah.edu.ni/files/uploads/biblioteca/880.pdf

Galeano, H.(2015). ¿POR QUÉ EMPRENDER COMO PERSONA FÍSICA O MORAL?. 06 marzo 2018, de Expansión CNN Sitio web: https://expansion.mx/opinion/2015/11/10/el-dilema-de-emprender-como-personafisica-o-persona-moral

López, J. (2016). Fracasan en México 75\% de emprendimientos. 02 febrero 2018, de El Financiero Sitio web: http://www.elfinanciero.com.mx/empresas/fracasan-en-mexico75-de-emprendimientos.html 
Año 11.

Frontera

Núm. 27
Revista de Investigación

Académica sin

ISSN: 2007-8870

\section{http://revistainvestigacionacademicasinfrontera.com}

Mancilla, H. y Parra, J. (2013). Estadística Descriptiva e Inferencial I. PDF] México.

Matarranz, A. (2014). Visión de Producto: para qué sirve y cómo se construye. 02 febrero 2018, de Conversis Consulting - Marketing orientado a resultados para mercados tecnológicos. Sitio web: https://www.youtube.com/watch?v=rUy7JEBu0F4

Medina, H. (1998). El ingreso y el gasto como medida del bienestar de los hogares: una evaluación estadística. En: Segundo Taller Regional sobre Medición del Ingreso en las Encuestas de Hogares-LC/R. 1886-1998-p. 341-371.

Mejía, M. R. L., \& Hernández, S. M. (2010). Los Sistemas de Contabilidad de Costos en la PyME mexicana. Investigación y Ciencia, 18(47), 49-56.

Muñoz, M. (2017). ¿Cuántos empleos en México podrían ser reemplazados por robots?. 02 febrero 2018, de CNN Sitio web: http://cnnespanol.cnn.com/2017/03/21/cuantosempleos-en-mexico-podrian-ser-reemplazados-por-robots/

Pineda, A. (2018). México cae cuatro lugares en el Índice Global de Emprendimiento. Recuperado: 6 de marzo de 2018, de EXPANSION CNN Sitio web: https://expansion.mx/emprendedores/2018/01/09/mexico-cae-cuatro-lugares-en-elindice-global-de-emprendimiento

Ríos, M. (2014). Método de Diagnóstico para Determinar el Sistema de Costes en una Pyme. Un Caso de Estudio. RIGC, 12(24), 13-22.

Rocha, H.. (2014). Los sistemas de costos y la eficiencia en las operaciones comerciales. 4 de abril de2018, de Revista Puntos Finos Thomson Reuters Sitio web: https://www.ccpm.org.mx/avisos/88-91Sistemas.pdf

Roldán, M. I. D., Montoya, L. F. G., \& Agudelo, J. A. O. (2009). Análisis de los sistemas de costos utilizados en las entidades del sector salud en Colombia y su utilidad para la toma de decisiones. Revista Digital del Instituto Internacional de Costos, (5), 495-525. 
Año 11.

Frontera

Núm. 27
Revista de Investigación

Académica sin

ISSN: 2007-8870

\section{http://revistainvestigacionacademicasinfrontera.com}

Secretaría de Economía. (2010). Microempresas. 05 febrero 2018, de Secretaría de Economía, México Sitio web: http://www.2006-2012.economia.gob.mx/mexicoemprende/empresas/microempresario?lang=es

Salas, A. (2013). Clasificación de los Costos. 09 enero de 2018, de Universidad Veracruzana Sitio web: https://www.uv.mx/personal/alsalas/files/2013/02/CLASIFICACION-DELOS-COSTOS.pdf

Spiegel, S. (2007). Políticas Macroeconómicas y de crecimiento. Nueva York, Publicación de las Naciones Unidas, Departamento de Asuntos Económicos y Sociales.

Vargas, I. (2010). El Empleado Multitareas ¿Bueno o Malo?. 02 febrero 2018, de EXPANSION CNN Sitio web: https://expansion.mx/mi-carrera/2010/04/23/empleotrabajo-sobrecarga-cnnexpansion

Vidales, P. (2013). Un México de emprendedores. El reto de la educación. Ponencia IMEF.

Vivanco, N. (2014). Tres de cada cuatro emprendedores financia su negocio con recursos propios. 6 de febrero de 2018, de La Tercera Sitio web: http://www2.latercera.com/noticia/tres-de-cada-cuatro-emprendedores-financia-sunegocio-con-recursos-propio 